\title{
Primer registro de pelícano (Aves: Pelecanidae) para el Mioceno tardío de la formación Pisco, Perú
}

Première découverte de pélican (Aves: Pelecanidae) au Miocène récent de la formation Pisco, Pérou

First fossil record of a pelican (Aves: Pelecanidae) from the Late Miocene of the Pisco Formation, Peru

\section{Alí J. Altamirano-Sierra}

\section{OpenEdition}

\section{Journals}

Edición electrónica

URL: https://journals.openedition.org/bifea/700

DOI: $10.4000 /$ bifea. 700

ISSN: 2076-5827

\section{Editor}

Institut Français d'Études Andines

\section{Edición impresa}

Fecha de publicación: 1 abril 2013

ISSN: 0303-7495

\section{Referencia electrónica}

Alí J. Altamirano-Sierra, «Primer registro de pelícano (Aves: Pelecanidae) para el Mioceno tardío de la formación Pisco, Perú», Bulletin de l'Institut français d'études andines [En línea], 42 (1) | 2013, Publicado el 24 junio 2014, consultado el 21 septiembre 2021. URL: http://journals.openedition.org/bifea/700 ; DOI: https://doi.org/10.4000/bifea.700

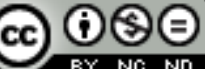

Les contenus du Bulletin de l'Institut français d'études andines sont mis à disposition selon les termes de la licence Creative Commons Attribution - Pas d'Utilisation Commerciale - Pas de Modification 4.0 International. 


\title{
Primer registro de pelícano (Aves: Pelecanidae) para el Mioceno tardío de la formación Pisco, Perú
}

\author{
Alí J. Altamirano-Sierra*
}

\begin{abstract}
Resumen
Se describe un os quadratum izquierdo fósil asignado al género Pelecanus proveniente de los yacimientos de nivel Montemar de la formación Pisco, en la costa centro-sur del Perú (región Arequipa). El fósil fue comparado con las especies actuales Pelecanus thagus y $P$. occidentalis, y con P. schreiberi del Plioceno de América del Norte. Este el primer registro de un pelícano fósil en toda la costa peruana. Se analizó además la paleo-avifauna de la formación Pisco, concluyendo que las especies actuales que son endémicas de la corriente peruana se encontraban presentes desde al menos el Mioceno tardío (6 Ma).
\end{abstract}

Palabras clave: Pelecanus, Mioceno tardío, formación Pisco, Perú

\section{Première découverte de pélican (Aves : Pelecanidae) au Miocène récent de la formation Pisco, Pérou}

\section{Résumé}

Nous décrivons un quadratum fossile gauche attribué au genre Pelecanus originaire du niveau de Montemar de la formation Pisco, côte centre-sud du Pérou (région d'Arequipa). Le fossile a été comparé avec les espèces actuelles Pelecanus thagus et $P$. Occidentalis, et avec $P$. Schreiberi du Pliocène de l'Amérique du Nord. C'est la première découverte fossile d'un pélican sur la côte péruvienne. La paléo-avifaune de la formation Pisco a également été analysée, ce que permet de conclure que les

* Departamento de Paleontología de Vertebrados, Museo de Historia Natural, Universidad Nacional Mayor de San Marcos, Av. Arenales 1256, Lima 14,Perú. E-mail: eraexcusa@yahoo.es 
espèces actuelles qui sont endémiques pour le Pérou actuel étaient présentes depuis au moins la fin du Miocène (6 Ma).

Mots-clés : Pelecanus, Miocène supérieur, formation Pisco, Pérou

\title{
First fossil record of a pelican (Aves: Pelecanidae) from the Late Miocene of the Pisco Formation, Peru
}

\begin{abstract}
A fossil quadrate bone referred to the genus Pelecanus is described. This fossil comes from the Montemar deposit of the Pisco Formation in the South-Central coast of Perú (Arequipa region). Comparison with the extant species Pelecanus thagus and P. occidentalis and the fossil P. schreiberi from the Pliocene of North America is also made. This is the first fossil pelican from the Peruvian coast. The fossil bird assemblage from the Pisco Formation is also analyzed, concluding that the species that are currently endemic to the Peruvian current were already present since at least the Late Miocene (6 Ma).
\end{abstract}

Key words: Pelecanus, Late Miocene, Pisco Formation, Peru

\section{INTRODUCCIÓN}

La familia Pelecanidae comprende ocho especies de aves marinas incluidas el actual género Pelecanus (Johnsgard, 1993). Dos de estas especies incluyen la costa peruana dentro de su área de distribución: el pelícano pardo Pelecanus occidentalis, visitante tropical no reproductivo en la costa norte $\left(\sim 3-6^{\circ} \mathrm{S}\right)$ y el pelícano peruano Pelecanus thagus, que anida desde los $5^{\circ} \mathrm{S}$ hacia el sur hasta los $27^{\circ}$ S, en Chile (Schulenberg et al., 2007: 54; Figueroa \& Stucchi, en revisión).

El registro más antiguo de Pelecanidae corresponde a Pelacanus sp. del Oligoceno temprano de Francia (Louchart et al., 2010: 16). Otras especies fósiles incluyen a Miopelecanus gracilis (Milne-Edwards, 1870) del Mioceno temprano de Francia (Cheneval, 1984: 68), M. intermedius (Fraas, 1870) del Mioceno medio de Alemania (Heizmann \& Hesse, 1995: 175), Pelecanus tirarensis (Miller, 1966) descubierta en los estratos del Mioceno medio y tardío de Australia (Rich \& Van Tets, 1981: 237) y P. schreiberi del Plioceno de América del Norte (Olson, 1999: 503-504).

En el presente trabajo se describe un os quadratum fósil asignado a la familia Pelecanidae, proveniente de los sedimentos del nivel Montemar de la formación Pisco (Muizon \& DeVries, 1985: 550; Marocco \& Muizon, 1988: 107). 


\section{MATERIAL Y MÉTODOS}

El espécimen fósil en estudio, catalogado con el código MUSM 583 (fig. 1), fue encontrado y colectado por Rodolfo Salas-Gismondi, en julio del 2005 y está depositado en la colección del Departamento de Paleontología de Vertebrados del Museo de Historia Natural de la Universidad Nacional Mayor de San Marcos (MUSM-UNMSM), Lima (Perú). Se trata de un os quadratum izquierdo fragmentario, con el processus oticus erosionado y el processus orbitalis incompleto (figs. 2A, C, E y G, 3 A y B).

Se determinaron las características morfológicas distintivas del espécimen en estudio y se compararon con especímenes actuales de Pelecanus thagus y P. occidentalis depositados en el MUSM-UNMSM. Adicionalmente, se realizó la comparación con fotografías del paratipo y espécimen referidos a Pelecanus schreiberi, del Florida Museum of Natural History (UF), en Gainesville (Estados Unidos) e información publicada por Saiff (1978) sobre P. roseus y Pelecanus rufescens. Así como también con especímenes de Phalacrocorax bougainvillii, P. gaimardi, P. olivaceous, Sula variagata, S. nebouxii, fotografía de especímenes de Fregata magnificens (FMNH 339421) y Phaethon rubricauda (339438) pertenecientes a la colección del Florida Museum of Natural History. Para la descripción morfológica se siguió la terminología propuesta por Baumel \& Witmer (1993).

La comparación métrica del espécimen con otras especies de Pelecanus fue realizada mediante tres medidas tomadas con un calibrador y se expresan en $\mathrm{mm}$.

\section{1. Geología, localidad y edad}

La formación Pisco es una secuencia sedimentaria marina del Neógeno. Se extiende en la costa por más de 325 km entre las ciudades de Pisco, Ica, Nazca, Lomas y Yauca, en las regiones de Ica y Arequipa, en el centro-sur del Perú (fig. 1 A y B). Se estima que el espesor total de la sección de la formación Pisco es de $640 \mathrm{~m}$ (Brand et al., 2003). Se han podido separar hasta nueve localidades fosilíferas, las cuales tienen diferentes edades que abarcan desde el Mioceno medio (11-13 Ma) hasta el Plioceno tardío (2 Ma) (Muizon \& DeVries, 1985: 552553; Muizon et al., 2004: 389; Stucchi, 2007: 369-370). El espécimen en estudio (MUSM 583) proviene de los sedimentos del nivel Montemar (fig. 1C), localidad considerada como Mioceno tardío y con una antigüedad de aproximadamente 6 Ma (Muizon \& DeVries, 1985: 556; Marocco \& Muizon, 1988: 107).

\section{SISTEMÁTICA PALEONTOLÓGICA}

- Clase: Aves Linnaeus, 1758

- Orden: Pelecaniformes Sharpe, 1891

- Familia: Pelecanidae Rafinesque, 1815

- Género: Pelecanus Linnaeus, 1758 


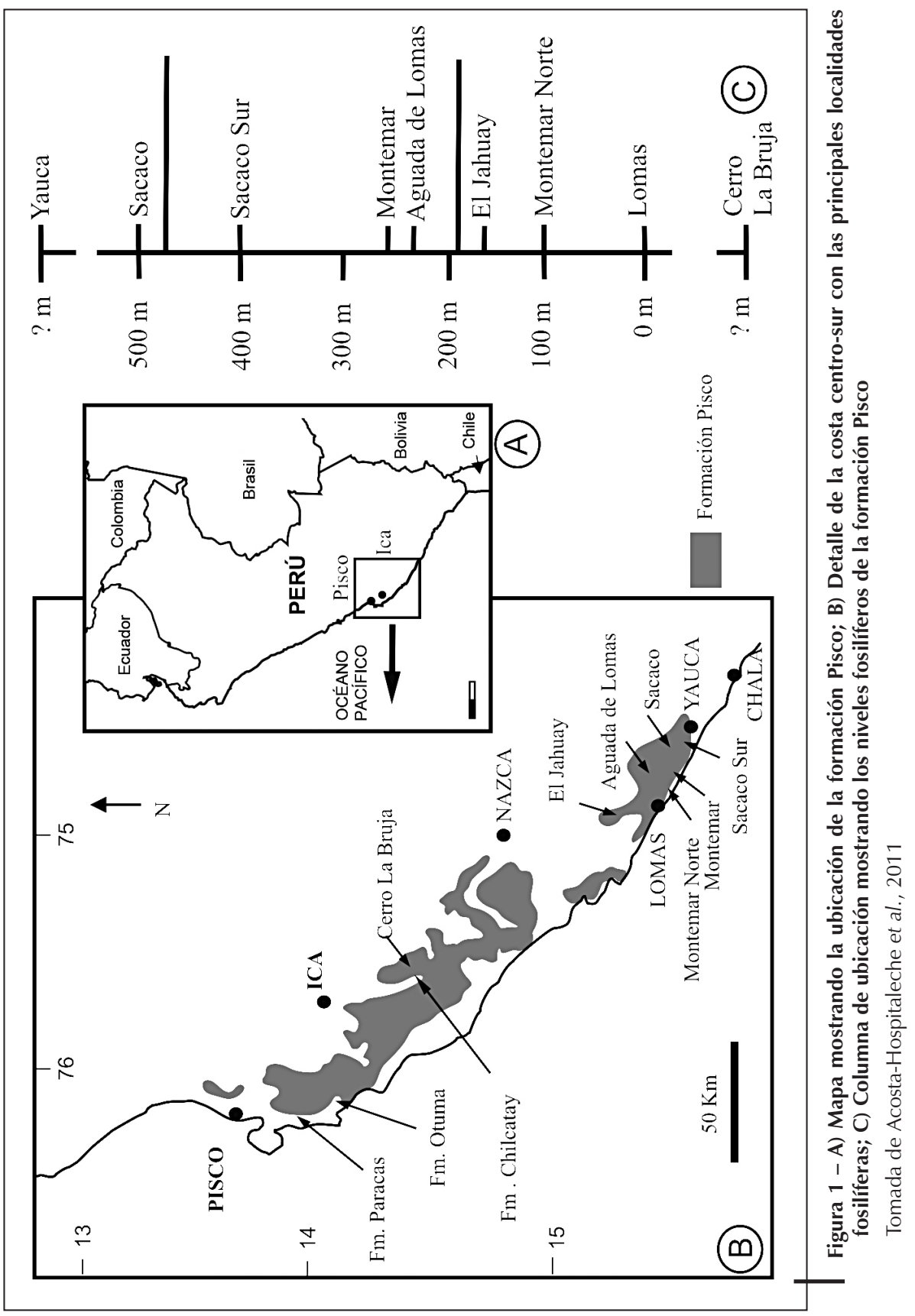


Primer registro de pelícano (Aves: Pelecanidae) para el Mioceno tardío de la formación Pisco, Perú



Figura 2 - Comparación del os quadratum de Pelecanus sp. (A, C, E y G, escala $15 \mathrm{~mm})$ y $P$. thagus (B, D, F y H, escala $20 \mathrm{~mm}$ )

Vistas A, B lateral, C, D medial, E, F craneal y G, H ventral 


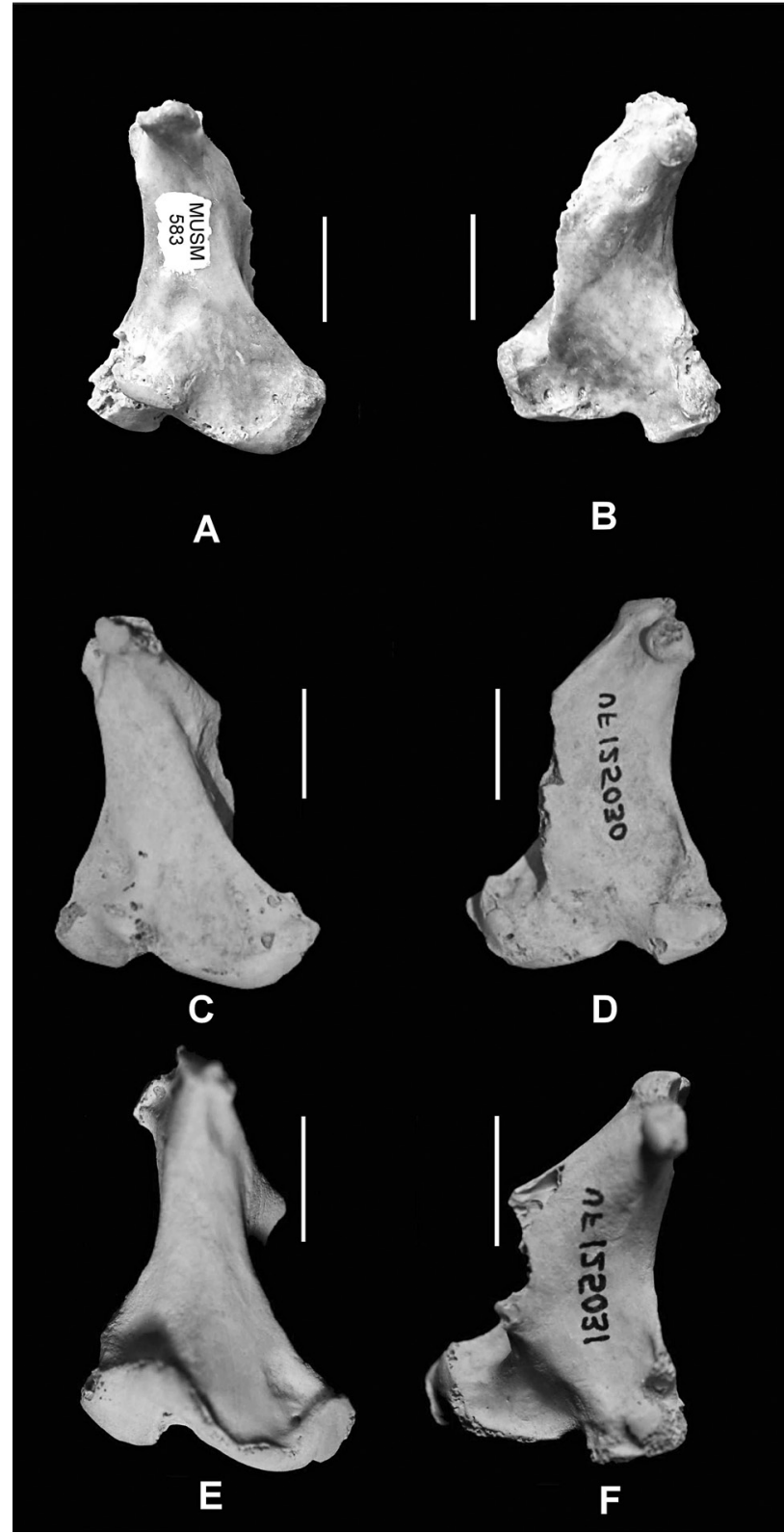

Figura 3 - Ossa quadrati fósiles de Pelecanus sp. MUSM 583 (A y B), Pelecanus schreiberi UF 125030 (C y D) (imagen invertida) y paratipo UF 125031 (E y F) (imagen invertida) Escala: $10 \mathrm{~mm}$ 
El espécimen, MUSM 583 está representado por un único hueso cuadrado asignado al género Pelecanus por la combinación de los siguientes caracteres:

- Corpus ossa quadrati comprimido antero-posteriormente (cuerpo más robusto y de forma cilíndrica en Fregata, Phaethon, Sula y Phalacrocorax).

- Ausencia de una fosa profunda en la cotyla quadratojugalis para la articulación con el condylus quadraticus del os quadratojugalis (fosa presente y profunda en Sula, Phaethon, Fregata y Phalacrocorax).

- Superficie ventral del processus mandibularis sin una fosa profunda que separa a los condyli pterigoideus y lateralis (superficie ventral con una fosa profunda en Phaethon, Sula, Fregata y Phalacrocorax).

Pelecanus sp. (Rich \& Van Tets, 1981: 236)

\section{1. Descripción del material MUSM 583}

El corpus ossa quadrati está pneumatizado y comprimido antero-posteriormente. La mayor parte del processus oticus donde están los capituli otica y squamosum se encuentran erosionados. En vista anterior, la base del processus orbitalis se curva hacia su extremo inferior; en su lado medial se encuentra un único foramen pneumaticum, del cual continúa una pequeña cresta que se conecta con el condylus pterygoideus; entre esta cresta y la base del processus orbitalis se forma una depresión, que es más profunda en el fósil que en especímenes observados de $P$. thagus. La presencia del foramen pneumaticum es variable, se encuentra presente en $P$. roseus y $P$. rufescens (Saiff, 1978: 328), pero tanto en $P$. occidentalis como en $P$. thagus su presencia es variable.

En vista ventral, el condylus pterygoideus tiene una forma alargada lateromedialmente. Entre los condyli pterygoideus y lateralis, se forma una leve depresión. En vista caudal, el condylus lateralis está dirigido ventro-lateralmente y el corpus ossa quadrati tiene una compresión latero-medial. En vista lateral, la cotyla quadratojugalis tiene una superficie convexa y no presenta una fosa para la articulación con el os quadratojugalis.

\section{DISCUSIÓN}

En América del Sur, fósiles asignados a la familia Pelecanidae han sido cuestionados. Liptornis hesternus (Ameghino, 1895), es considerado como Aves incertae sedis por Olson (1985: 201) y Warheit (2002: 40). Sin embargo, recientemente Cenizo \& Agnolín (2010: 498-499), Degrange et al. (en prensa) y Diederle (2011) demuestran que este taxón es válido y corresponde a un Anhingidae. El espécimen MUSM 583 demuestra la presencia de la familia Pelecanidae en el Mioceno tardío y representa el único y más antiguo registro fósil del género Pelecanus en América del Sur.

Las dimensiones de MUSM 583 son mayores que las de Pelecanus occidentalis y sugieren un tamaño similar a los especímenes de menores dimensiones del 
pelícano peruano $P$. thagus (tabla 1). En América del Norte en las localidades de Lee Creek Mine, Carolina del Norte y del Centro de Florida se ha descrito P. schreiberi (Olson, 1999), para el Plioceno temprano de las formaciones Yorktown y Bone Valley. El paratipo (UF 125031) y un os quadratum referido a esta especie (UF 125030) se diferencian de MUSM 583 por la forma más aguzada del condylus pterygoideus, como se aprecia claramente en su vista lateral (fig. 3). Las dimensiones del cuadrado presentadas por Olson (1999: 506) (ver tabla 1) son menores en longitud que las de MUSM 583, mientras que el largo de la articulación mandibular es mayor en $P$. schreiberi. Las comparaciones con otras especies fósiles de pelícanos (Miopelecanus gracilis, Miopelecanus intermedius y Pelecanus tirarensis) no fueron posibles debido a que no se conservó el hueso cuadrado en estas especies.

Tabla 1 - Medidas de los huesos cuadrados de MUSM 583, Pelecanus occidentalis $(n=2)$ y Pelecanus thagus $(\mathrm{n}=5)$. Las medidas de Pelecanus schreiberi fueron tomadas de Olson (1999)

\begin{tabular}{|c|c|c|c|}
\hline Especímenes & $\begin{array}{c}\text { Altura máxima } \\
(\mathbf{m m})\end{array}$ & $\begin{array}{c}\text { Ancho máximo } \\
(\mathbf{m m})\end{array}$ & $\begin{array}{c}\text { Longitud de } \\
\text { la articulación } \\
\text { mandibular }(\mathbf{m m})\end{array}$ \\
\hline Pelecanus sp. MUSM 583 & 36,49 & 12,5 & 23,84 \\
\hline Pelecanus schreiberi & 33,0 & - & 25,0 \\
\hline Pelecanus occidentalis & $34,97-35,24$ & $11,6-11,7$ & $22,8-22,7$ \\
\hline Pelecanus thagus & $42,75-36,78$ & $14,84-11,49$ & $28,41-23,32$ \\
\hline
\end{tabular}

\section{1. Paleoecología}

De acuerdo con Keller et al. (1997: 129), en base a datos micropaleontológicos, durante el Cretácico tardío una corriente de aguas frías fluctuaba frente a las costas del Ecuador y Perú de manera similar a la Corriente Peruana actual. Los sedimentos biogénicos de las cuencas de antearco (Sechura y Pisco) del margen continental del Perú, son consistentes con episodios de alta producción primaria y fenómenos de afloramientos durante los últimos 40 Ma (Dunbar et al., 1990: 253), La secuencia sedimentaria miocénica y pliocénica de la formación Pisco es consistente con un clima árido o semiárido, el cual estuvo presente desde el Mioceno medio (Alpers \& Brimghall, 1988: 1640), aunque otros autores consideran que habría iniciado en el Jurásico (Hartley et al., 2005: 422). Desde el Mioceno medio hasta el Plioceno temprano la costa peruana estaba formada por bahías protegidas y arrecifes; frente a ellas existían cadenas de islas que mantenían separada la costa del mar abierto (Marocco \& Muizon, 1988: 111; Muizon \& DeVries, 1985: 558). La temperatura de esta corriente durante el Mioceno y Plioceno se estimó entre 13,0-17,2 ${ }^{\circ} \mathrm{C}$ (Amiot et al., 2008: 91), muy similar a la que presenta la actual Corriente Peruana en el Centro-Sur del Perú (Gunther, 1936: 148). 
La paleo-avifauna del Mioceno-Plioceno de la formación Pisco incluye dos especies de Diomedeidae, Procellariidae, seis especies de Sulidae, dos especies de Phalacrocorax, cinco especies de Spheniscus, Pelagornithidae (Stucchi, 2010: 35) y Pelecanus sp. (este texto).

Esta paleo-avifauna marina muestra una afinidad muy cercana a la asociación de especies que habitan actualmente la costa peruana. Algunas especies como: Sula aff. variegata y Phalacrocorax aff. bougainvillii se conocen para el Mio-Plioceno (Stucchi, 2003: 79; Urbina \& Stucchi 2005: 43). Phalacrocorax bougainvillii también es conocida de los depósitos pleistocénicos del área de Talara en el norte del país (Campbell, 1979: 22).

La Corriente Peruana actual alberga un ecosistema muy particular el cual depende de la alta producción primaria, la cual a su vez depende de una serie de procesos meteorológicos y oceanográficos como el afloramiento, la termoclina y las ondas ecuatoriales (Arntz \& Fahrbach, 1991: 19-33). En este ecosistema habitan 93 especies de aves marinas entre Pelecaniformes, Procellariiformes, Sphenisciformes y Charadriiformes (Spear \& Ainley, 2008: 139-140). Once de estas especies son aves endémicas (Crawford et al., 2006: 553), de las cuales Phalacrocorax bougainvillii, Sula variegata y Pelecanus thagus se asocian ecológicamente en un solo grupo de acuerdo a su tipo de alimentación (Spear \& Ainley 2008: 133). Todas estas especies se alimentan principalmente de la anchoveta (Engraulis ringens) (Crawford et al., 2006: 553). Los fósiles asignados a las especies, Phalacrocorax aff. bougainvillii y Sula aff. variegata, presentan la misma morfología que las actuales especies endémicas de la Corriente Peruana. La presencia de Pelecanus sp. junto con estas especies sugiere que el mismo nicho ecológico que ocupan estas aves en la actualidad fueron ocupadas por estas aves desde finales del Mioceno (6 Ma).

\section{CONCLUSIÓN}

En base a sus características morfológicas, se concluye que MUSM 583 pertenece al género Pelecanus. Su procedencia estratigráfica demuestra que el regristro presentado aquí corresponde al representante más antiguo de este género en América del Sur. El ambiente en que se desarrolló la paleo-avifauna de la formación Pisco durante el Mioceno y Plioceno era muy similar al actual. Este escenario fue propicio para el desarrollo de especies endémicas del ecosistema de la Corriente Peruana desde al menos el Mioceno tardío (6 Ma) hasta la actualidad.

\section{Agradecimientos}

A los biólogos Marcelo Stucchi, Rodolfo Salas-Gismondi y a la Dra. Julia Clarke por la revisión de las versiones precedentes de este artículo. Al biólogo Julio C. Reyes por el apoyo brindado durante la investigación. A los doctores Daniel Ksepka y Dana Ehret por las publicaciones y fotografías que ayudaron a enriquecer este trabajo. 


\section{Referencias citadas}

ACOSTA-HOSPITALECHE, C., ALTAMIRANO-SIERRA, A. \& STUCCHI, M., 2011 Variaciones morfológicas del tarsometatarso de los pingüinos (Aves, Sphenisciformes) de la secuencia estratigráfica de la formación Pisco (Mio-Plioceno), Perú. Revista Mexicana de Ciencias Geológicas, 28 (2): 290-300.

ALPERS, C. \& BRIMHALL, G., 1988 - Middle Miocene climatic change in the Atacama Desert, northern Chile: Evidence from supergene mineralization at La Escondida. Geological Society of America Bulletin, 100: 1640-1656.

AMIOT, R., GÖHLICH, U., LÉCUYER, C., MUIZON, C., CAPPETTA, H., FOUREL, F., HÉRAN, M.-A. \& MARTINEAU, F., 2008 - Oxygen isotopes of phosphate from Middle Miocene-Early Pliocene marine vertebrates from Peru. Palaeogeography, Palaeoclimatology, Palaeoecology, 264: 85-92.

ARNTZ, W. E. \& FAHRBACH, E., 1991 - El Niño: Experimento climático de la naturaleza, 309 pp.; Mexico, D. F.: Fondo de Cultura Económica.

BAUMEL, J. \& WITMER, L., 1993 - Osteologia. In: Handbook of avian anatomy: Nomina anatomica avium (J. Baumel, A. King, J. Breazile, H. Evans \& J. Vanden Berge, eds.): 45-132; Cambridge: Publications of the Nuttal Ornithological Club.

BRAND, L., URBINA, M., CARVAJAL, C. \& DEVRIES, T., 2003 - Stratigraphy of the Miocene/ Pliocene Pisco Formation in the Pisco Basin. Geological Society of America, 2003 Annual meeting. Geological Society of America, Seattle. Abstracts with Programs, Vol. 35, n. ${ }^{\circ}$ 6: 160.

CAMPBELL, K., 1979 - The non-passerine Pleistocene avifauna of the Talara Tar Seeps, Northwestern Peru, 203 pp.; Toronto: Royal Ontario Museum.

CENIZO, M. \& AGNOLIN, F., 2010 - The southern most record of the Anhingidae and a new basal species of Anatidae (Aves) from the lower-middle Miocene of Patagonia, Argentina. Alcheringa, 34: 493-514.

CHENEVAL, J., 1984 - Les oiseaux aquatiques (Gaviiformes a Ansériformes) du gisement Aquitanien de Saint-Gérand-Le-Puy (Allier, France): Révision systématique. Palaeovertebrata, 14 (2): 33-115.

CRAWFORD, R., GOYA, E., ROUX, J.-P. \& ZAVALAGA, C., 2006 - Comparison of assemblage and some life-history traits of seabirds in the Humboldt and Benguela systems. South African Journal of Marine Science, 28 (3-4): 553-560.

DEGRANGE, F., NORIEGA, J. \& ARETA, J., en prensa - Diversity and paleobiology of the Santacrucian birds. In: Early Miocene paleobiology in Patagonia: High-Latitude paleocommunities of the Santa Cruz formation (S. Vizcaino, R. Kay \& S. Bargo, eds.): 138-155; Cambridge: Cambridge University Press.

DIEDERLE, J., 2011 - El status sistemático de Liptornis hesternus Ameguino, 1895 (Aves: Pelecaniformes) de la Formación Santa Cruz, Patagonia. Resumen IV Congreso Latinoamericano Paleontología de Vertebrados, San Juan Argentina, 2011.

DUNBAR, R., MARTY, R. \& BAKER, P., 1990 - Cenozoic marine sedimentation in the Sechura and Pisco basins, Peru. Palaeogeography, Palaeoclimatology, Palaeoecology, 77: 235-261.

FIGUEROA, J. \& STUCCHI, M., en revisión - Registros de reproducción más septentrionales del Pelícano Peruano Pelecanus thagus y el Ostrero Negro Haematopus ater. Boletín Sociedad Antioqueña de Ornitología - Colombia. 
Primer registro de pelícano (Aves: Pelecanidae) para el Mioceno tardío de la formación Pisco, Perú

GUNTHER, E. R., 1936 - A report on oceanographical investigations in the Peru coastal current. Discovery Reports, 13: 107-276.

HARTLEY, A., CHONG, G., HOUSTON, J. \& MATHER, A., 2005 - 150 Million years of climatic stability: Evidence from the Atacama desert, northern Chile. Journal of the Geological Society of London, 162: 421-424.

HEIZMANN, E. P. \& HESSE, A., 1995 - Die Mittelmiozän vogel-und säugetierfaunen des Nördlinger Ries (MN6) und des Steinheimer Beckens (MN7) - ein vogel vergleich. Courier Forschungsinstitut Senckenberg, 181: 171-185.

JOHNSGARD, P., 1993 - Cormorants, darters and pelicans of the world, 445 pp.; Washington \& London: Smithsonian Institution Press.

KELLER, G., ADATTE, T., HOLLIS, C., ORDOÑEZ, M., ZAMBRANO, I., JIMÉNEZ, N., STINNESBECK, W., ALEMAN, A. \& HALE-ELRICH, W., 1997 - The Cretaceous/ Tertiary boundary event in Ecuador: reduced biotic effects due to eastern boundary current setting. Marine Micropaleontology, 31: 97-133.

LOUCHART, A., TOURMENT, N. \& CARRIER, J., 2010 - The earliest know pelican reveals 30 million years of evolutionary stasis in beak morphology. Journal of Ornithology, 152 (1): 15-20.

MAROCCO, R. \& MUIZON, C., 1988 - Los vertebrados del Neógeno de la costa Sur del Perú: Ambiente sedimentario y condiciones de fosilización. Bulletin de I'Institut Français d'Études Andines, 17 (2): 105-117.

MUIZON, C. \& DEVRIES, T., 1985 - Geology and paleontology of the late Cenozoic marine deposits in the Sacaco area (Peru). Geologische Rundschau, 74 (3): 547-563.

MUIZON, C., MCDONALD, G., SALAS, R. \& URBINA, M., 2004 - The youngest species of the aquatic sloth Thalassocnus and a reassessment of the relationships of the Nothrothere sloths (Mammalia: Xenarthra). Journal of Vertebrate Paleontology, 24 (2): 387-397.

OLSON, S., 1985 - The fossil record of birds. In: Avian biology vol. VIII (D. S. Farner, J. R. King \& K. C. Parker, eds.): 79-256; Nueva York: Academic Press.

OLSON, S., 1999 - A new species of pelican (Aves: Pelecanidae) from the Lower Pliocene of North Carolina and Florida. Proceedings of the Biological Society of Washington, 112 (3): 503-509.

RICH, P. \& VAN TETS, G., 1981 - The fossil pelicans of Australasia. Records of the South Australian Museum, 18 (12): 235-264.

SAIFF, E. I., 1978 - The middle ear of the skull of birds: the Pelecaniformes and Ciconiiformes. Zoological Journal of the Linnean Society, 63: 315-370.

SCHULENBERG, T., STOTZ, D. F., LANE, D. F., O’ NEILL, J. \& PARKER III, T. A., 2007 - Birds of Peru, 656 pp.; Princenton: Princenton University Press.

SPEAR, L. B. \& AINLEY, D. G., 2008 - The seabird community of the Peru current, 19801995, with comparison with other eastern boundary currents. Marine Ornithology, 36: $125-144$.

STUCCHI, M., 2003 - Los piqueros (Aves: Sulidae) de la formación Pisco. Boletín de la Sociedad Ceológica del Perú, 95: 75-91.

STUCCHI, M., 2007 - Los pingüinos fósiles de la formación Pisco (Neógeno), Perú. In: 4th European Meeting on the paleontology and Stratigraphy of Latin America (E. DíazMatinez \& I. Rábano, eds.): 367-373; Madrid: Tres Cantos.

STUCCHI, M., 2010 - Alcances sobre la metodología de estudio en Paleontología de Vertebrados. Boletín de la Sociedad Ceológica del Perú, 104: 33-40. 
URBINA, M. \& STUCCHI, M., 2005 - Los cormoranes (Aves: Phalacrocoracidae) del MioPlioceno de la formación Pisco, Perú. Boletín de la Sociedad Geológica del Perú, 99: 41-49.

WARHEIT, K., 2002 - The seabird fossil record and the role of paleontology understanding seabird community structure. In: Biology of Marine Birds (E. A. Schreiber \& J. Burger, eds): 17-56. 